Communications in Physics, Vol.20, No. 1 (2010), pp. 83-90

\title{
DEMONSTRATE THE DOUBLE NEGATIVE BEHAVIOR OF METAMATERIAL USING THE EFFECTIVE MEDIUM THEORY
}

\author{
NGUYEN THANH TUNG \\ Institute of Engineering Physics, \\ Military Academy of Science and Technology, Vietnam \\ and \\ Quantum Photonic Science Research Center, \\ Hanyang University, Seoul, 133-791 Korea \\ PHAM VAN HOAI, NGUYEN TRUNG BINH \\ Institute of Engineering Physics, \\ Military Academy of Science and Technology, Vietnam \\ LE VAN HONG, VU DINH LAM \\ Institute of Materials Science, VAST \\ Y. P. LEE \\ Quantum Photonic Science Research Center, \\ Hanyang University, Seoul, 133-791 Korea
}

\begin{abstract}
We fabricated the electric and magnetic metamaterial, and measured their transmission spectra. The electromagnetic response of the combined structure, which consists of cut-wire pair and continuous wire, are experimentally and numerically studied in the microwave frequency regime. The simulations are performed in a qualitative agreement with experiment utilizing the transfer-matrix method. The double negative behavior of combined structure is observed and demonstrated using the effective medium theory.
\end{abstract}

\section{INTRODUCTION}

Recently, the naturally available range of the matter-wave interaction is extended by the appearance of artificial-class materials, the so-called metamaterials. As common understanding, complex magnetic permeability and dielectric permittivity are two fundamental parameters that describe the response of matter to external electric and magnetic fields. With the brought-forth scenario, we can control the effective permittivity and permeability by tuning the structural parameters, and hence, obtain the desired values at any interested frequency. Consequently, the novel properties of metamaterials such as negative refraction, negative phase velocity, and the left-handed rule of $k, E$ and $H$ vectors, which are observable in the simultaneously negative permittivity and permeability medium, initially predicted by Veselago [1]. More than 30 years long after, the first experimental demonstration of the existence of a double negative metamaterial (DNM) was realized using the combination between split-ring resonator (SRR) and continuous wire based on Pendry's suggestion [2]. Later, the negative refractive index, which was the 
most challenging property of DNM, was shown in Shelby's experiment [3], The specific properties of DNMs make them become more and more interesting for the physical and technological applications, such as super lens [4], electromagnetic cloaking [5] and perfect absorber [6]. Therefore, the existences and the characteristics of DNMs is unfailingly a spellbound area, especially for DNM working at optical frequencies.

It well known that the magnetic metamaterials provide a negative permeability and the electric metamaterials yield a negative permittivity. Combining the magnetic and the electric components is the most common way to obtain the DNMs. While the negative permittivity can be simply archived by a periodic array of continuous wires [7], the desirable negative permeability is the choice in several magnetic resonators [8-10]. Among them, the cut-wire pair (CWP) structure, which comes from the flattening of two-gap SRR, has attracted a considerable attention due to its easily fabricating and high applicability [11-13]. Beside the ebullience in searching for the suitable magnetic and electric systems [14-16], the realization of the existence of DN behavior is still an important work in studying metamaterials [10,17]. Normally, the DN behavior cannot be obviously determined as usual by only employing the scattering parameters. It could be observed, but usually together with the double positive one (right-handed behavior), and both of them exhibit the transmission, which might separate by a certain amount of frequency [18]. In this study, we experimentally and numerically study the DN behavior of the combined structure, which consists of a periodic array of CWPs and continuous wires. The simulated results are in a qualitative agreement with experiments. The existence of DN behavior is essentially demonstrated using effective medium theory [19].

\section{NUMERICAL AND EXPERIMENTAL SETUP}

In our numerical calculation, we utilized the transfer-matrix method (TMM). The original algorithm of the TMM, based on the scattering formalism, was described by MacKinnon and Kramer [20] to numerically reassert the scaling theory of localization. Later, it has been generalized to solve the problems of electromagnetic waves in the inhomogeneous medium by Pendry et al. [21]. The TMM not only becomes the well-known standard tool for the numerical analysis on the transport properties of disordered electronic structures [22], but also is proven to be useful for the numerical simulation on photonic band-gap materials [23]. Recently, a DNM structure consisting of the SRRs and the continuous wires has been studied by Markos et al. [24, 25] using an improved TMM. The agreement between experimental measurements and theoretical predictions for the transmission properties of SRRs structure was confirmed. In the TMM, the sample is considered as a scatterer for the incoming wave and is characterized by four parameters: transmission of the wave from left to right $t_{+}$, from right to left $t_{-}$, and the reflection from right to right $r_{+}$and left to left $r_{-}$. The corresponding scattering matrix $S$ comes to be

$$
S=\left(\begin{array}{ll}
t_{+} & r_{+} \\
r_{-} & t_{-}
\end{array}\right)
$$

which determines the amplitudes of outgoing wave $B$ and $C$ in terms of the amplitudes of incoming wave $A$ and $D$, namely, 


$$
\left(\begin{array}{c}
C \\
B
\end{array}\right)=S\left(\begin{array}{c}
A \\
D
\end{array}\right)
$$

It can be rewritten into the transfer matrix form as

$$
\left(\begin{array}{l}
D \\
C
\end{array}\right)=T\left(\begin{array}{c}
B \\
A
\end{array}\right)
$$

where $T$ is the transfer matrix

$$
T=\left(\begin{array}{ll}
t_{-}^{-1} & -t_{-}^{-1} r_{-} \\
r_{+} t_{-}^{-1} & t_{+}-r_{+} t_{-}^{-1} r_{-}
\end{array}\right)
$$

which determines the outgoing electromagnetic fields on one side of structure from the incident fields [23]. One of the most important properties of the transfer matrix is to obey the composition law. If the sample consists of two substructures, then the total transfermatrix of the whole system can be calculated by multiplying the transfer matrices of substructures as below

$$
T_{12}=T_{2} T_{1}
$$

The total transfer matrix has the same form as the primary one. The composition law enables us to calculate the transmission of complicated structures [23]. In the numerical simulation, the total volume of the system is divided into small cells, and the fields in each cell are coupled to those in the neighboring one. The unit cell is discretized according to the original algorithm described in [24, 25]. For each point of the lattice, four components of the electromagnetic field $E_{x}, E_{y}, H_{x}$ and $H_{y}$ are calculated. Detail about the TMM was presented in [26].

A combined structure consists of a periodic array of unit cells and each unit cell contains a pair of cut-wire and continuous wires in parallel direction. Fig. 1(a) shows a unit cell of combined structure which was used in simulation. In this study, the incident wave propagates along the $z$ direction, while the $H$ and $E$ fields are assumed to be polarized along the $x$ and $y$ axis, respectively.

Fig. 1(b) defines the structural parameters of combined structure, where $L_{x}, L_{y}$ and $L_{z}$ are lattice constants in the $x, y$ and $z$ directions, respectively. The thicknesses of metal pattern and dielectric spacer are denoted by $t_{c}$ and $t_{s}$ while the width and the length of cut-wire are $w$ and $l$. The unit cell is divided into $N_{x} \times N_{y} \times N_{z}$ mesh points so that each mesh point is regarded as a homogeneous medium. We call the minimum unit length $\delta$ : the homogeneous discretization or the discrected length. All length parameters are given as integer multiples of $\delta$. In our model, $L_{x}=\delta \times N_{x}, L_{y}=\delta \times N_{y}$ and $L_{z}=\delta \times N_{z}$. The thickness of metal pattern is assume to be equal to the minimum unit length $\left(t_{c}=\right.$ $\delta)$ in this simulation, which is nearly ten times larger than the actual thickness $(0.036$ $\mathrm{mm})$. Although, we did not expect that the thickness considerably influences on the electromagnetic properties of the structure, in fact this induces a slight difference between simulation data and experimental results (see Fig. 2). However, it is still reasonable because the metal used in experiments is copper, which exhibits a high conductivity at the frequency of interest. The complex dielectric constant of spacer for simulation is kept 

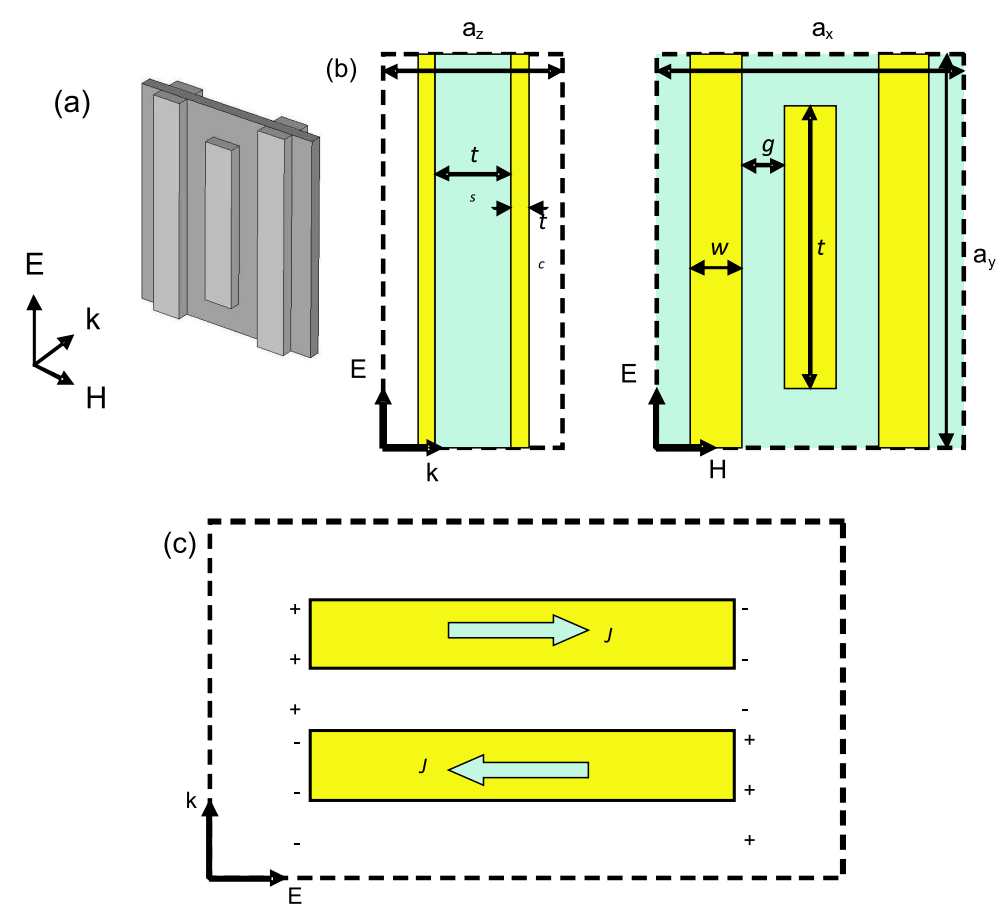

Fig. 1. (a) A unit cell of combined structure. (b) Geometry of a unit cell of combined structure. (c) The loop current inside a pair of cut-wires, here $\mathrm{J}$ is current vector.

to be equal to the experimental value, $5.1+i .0 .31$. It is assumed that the embedded reference medium is vacuum.

For the experiment, the cut-wire pair and the combined structure were fabricated using the conventional printed circuit board (PCB) process with $36 \mu \mathrm{m}$-thick copper patterns on both sides of a dielectric PCB spacer with a thickness of $0.4 \mathrm{~mm}$. Further details on the fabrication of the cut-wire-pair as well as the combined structure are given in Ref. 18. The length and the width of cut-wire pair are kept to be 5.6 and $1.2 \mathrm{~mm}$, respectively, and the width of the continuous wire is $1.2 \mathrm{~mm}$. We performed the transmission measurements in free space using a Hewlett-Packard E8362B network analyzer connected to the microwave standard-gain horn antennas.

\section{RESULTS AND DISCUSSIONS}

One fundamentally developed method used for the determination whether a metamaterial is single-, double- negative or positive is using effective medium theory [19]. Since the DN behavior is composed by a negative permeability $(\mu<0)$ and a negative permittivity $(\varepsilon<0)$ at the same frequency, we start by considering the transmission spectrum of $\mu$-negative medium. The magnetic resonance of CWP resonator provides the negative permeability, comes from the oscillation of circular current inside a pair of cut-wires [27], as shown in Fig. 1(c). The magnetic resonance can be determined using LC equivalent 
circuit with the inductance of cut-wires and the capacitance of gap between a pair of cut-wires. Short-circuiting at the end of the cut-wire-pair structure through the dielectric spacer is known to eliminate the capacitor of the cut-wire pair, and as a consequence, lead to the magnetic resonance vanishing. By this way, we can construct another effective medium, the so-called shorted CWP medium, without magnetic resonance, in other words, non negative permeability medium. It means that we will observe the $\mu$-negative band gap made of magnetic resonance in transmission spectrum of CWP medium. This band gap will be entirely disappeared in the shorted CWP medium.

For the negative permittivity, it is well known that the $\varepsilon$-negative medium can be obtain below plasma frequency using the periodic array of continuous wire [28]. The composite medium of CWPs and continuous wire can exhibit the DN behavior. However, the shorted combined structure consisting of the shorted CWPs and continuous wires does not exhibit the DN behavior. The transmission spectra of two composite media are able to qualitatively infer whether the medium is DN or not (see also Figs. 3 and 4). The predicted behaviors of combined structure using qualitatively effective medium theory were experimentally and numerically verified as shown in Fig. 3.

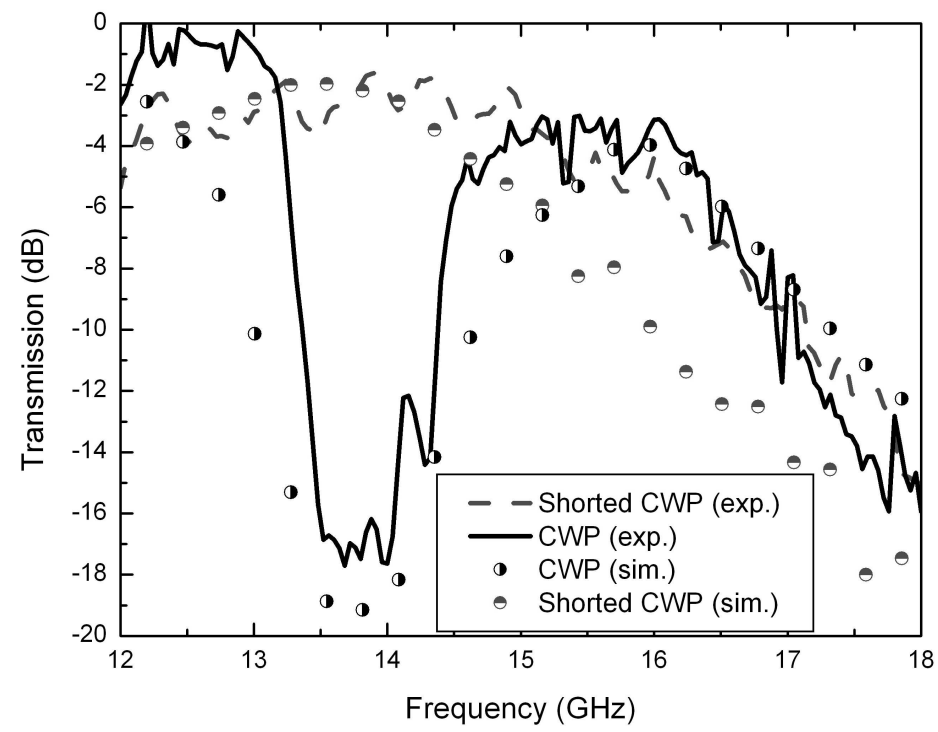

Fig. 2. The measured and the calculated transmission spectra of cut-wire pair and its shorted medium.

Fig. 2 presents the measured and calculated transmission spectra of CWPs and shorted CWPs media. In case of CWPs medium, the capacitance between cut-wires in a pair that are formed by circular currents inducing from magnetic field that resembles a magnetic resonance band. When shorting the capacitance, the resonating by magnetic field is eliminated, and hence, the band gap of effective medium is disappeared. By employing this method, we can thereby infer merely from transmission spectra whether the stop band exhibits the negative permeability or not. As shown in Fig. 2, the band gap of the CWP 
medium between 13.4 and $14.65 \mathrm{GHz}$ does not appear in case of shorted one. Therefore, this band gap exhibits the negative permeability. The other band gap starting from 17 $\mathrm{GHz}$ is corresponding to electric resonance, still be available even shorting CWPs. A good agreement between measurements and simulations is achieved.

Fig. 3 reveals the transmission of only continuous wires, CWPs and combined structure medium as a function of frequency. Again, a good agreement between measurements and simulations is achieved. Clearly, the continuous wires act as a $\varepsilon$-negative plasmon system with a plasma frequency, which is higher than the measured frequency range. As aforementioned, the band gap between 13.4 and $14.65 \mathrm{GHz}$ of CWP media exhibits the negative permeability. From the transmission spectra in Fig. 3, we assert that the transmission peak $\sim 13.9 \mathrm{GHz}$ is the result of the DN parameters $(\varepsilon$ and $\mu<0)$, in other words, indicating the left-handed behavior.
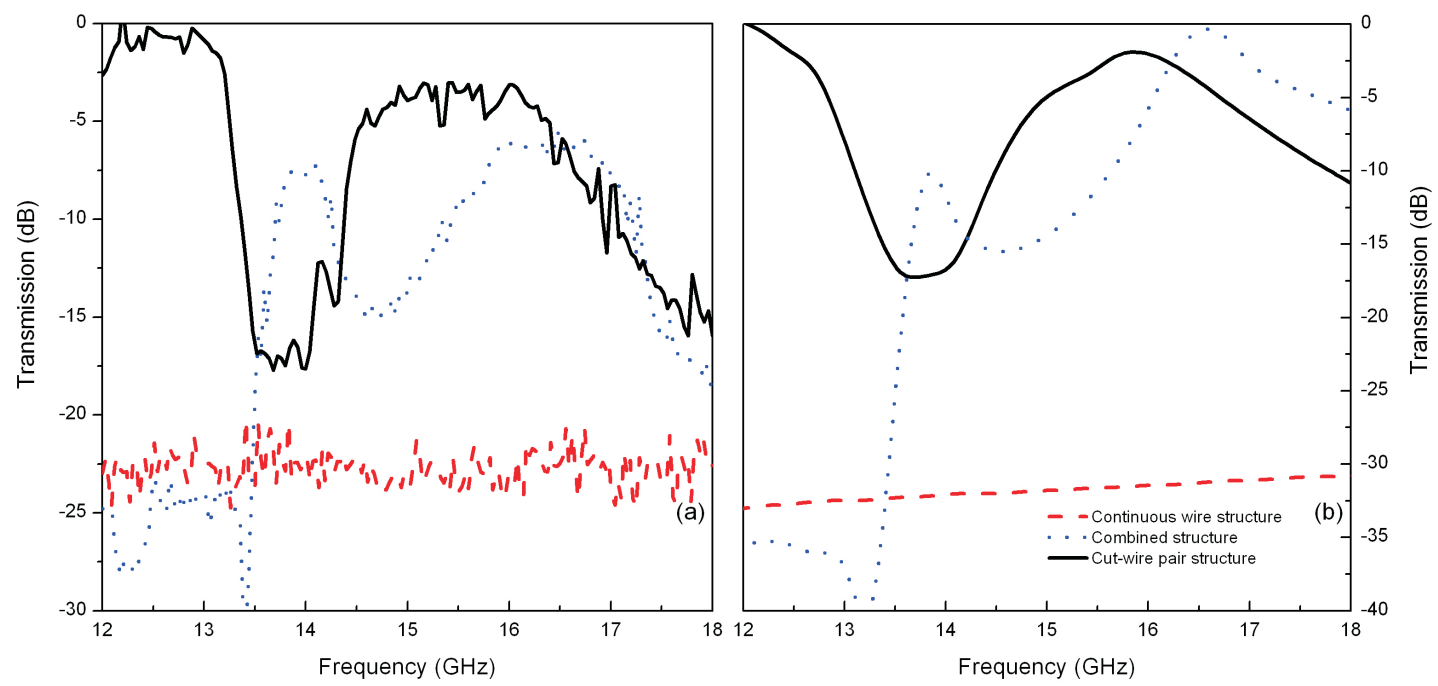

Fig. 3. (a) Experimental transmission spectra of continuous wires, cut-wire pair and combined structure, and (b) the simulated ones.

In addition, we observed another transmission band of combined structure from 15.7 to $17.1 \mathrm{GHz}$, which exhibits the double positive parameters $(\varepsilon$ and $\mu>0)$. The understandable reason for this phenomenon is that the combined structure consists of CWPs and continuous wires, which exhibits a lower total plasma frequency than continuous wire medium only [19]. Furthermore, the cut-wire pair does not provide a magnetic resonance in frequency range from 15.7 to $17.1 \mathrm{GHz}$, and also the effective permittivity of combined structure is positive. Therefore, both the effective permittivity and permeability are positive in this frequency range, result in obtaining the double positive. This explanation was also reaffirmed by the numerical calculation as shown in Fig. 4, which presents the transmission of combined and shorted combined structures.

The shorted combined structure consists of the shorted cut-wire pair and the continuous wire, which provides important information in determining the effective plasma 


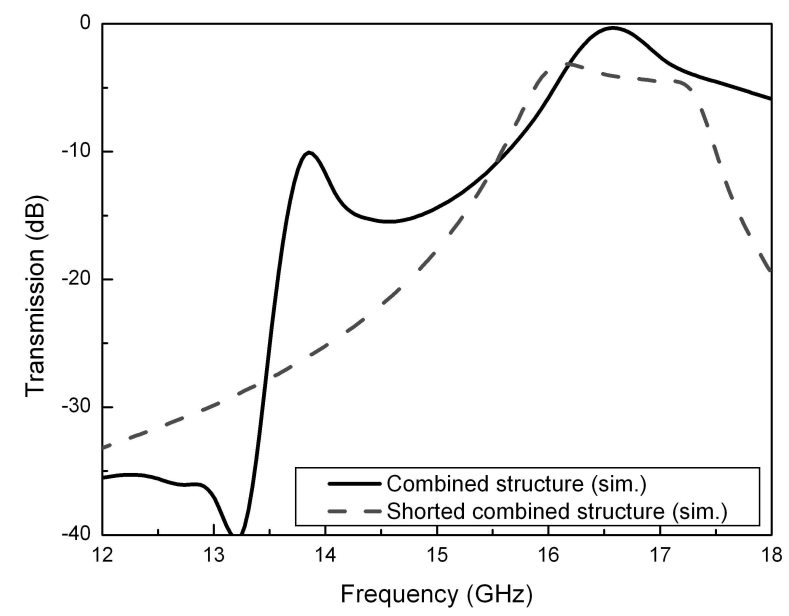

Fig. 4. The simulated transmission spectra of combined structure and shorted combined structure.

of the combined structure. As shown in Fig. 4, the shorted combined structure does not exhibit the DN transmission peak at $13.9 \mathrm{GHz}$, but the second transmission band still remains. It means that the total plasma frequency of the combined structure is around $15 \mathrm{GHz}$, hence, the second observed transmission band from 15.7 to $17.1 \mathrm{GHz}$ exposes the double positive behavior.

In conclusion, the DN behavior of a planar combined metamaterial operating at microwave frequency was investigated and demonstrated both experimentally and theoretically using the effective medium theory. The structures are fabricated via conventional photo-lithography technology. The responses of the electric and magnetic metamaterial were studied separately. All the experimental results are reasonably coincident with the numerical simulation using TMM.

\section{ACKNOWLEDGEMENTS}

This work was supported by the National Foundation for Science and Technology Development (NAFOSTED 103.02.36.09) through the Institute of Materials Science, Vietnam Academy of Science and Technology.

\section{REFERENCES}

[1] V. G. Veselago, Sov. Phys. Usp. 10 (1968) 509.

[2] J. B. Pendry, Phys. World 13 (2000) 27.

[3] R. A. Shelby, D. R. Smith, and S. Shultz, Science 292 (2001) 77.

[4] J. B. Pendry, Phys. Rev. Lett. 85 (2000) 3966.

[5] D. Schurig, J. J. Mock, B. J. Justice, S. A. Cummer, J. B. Pendry, A. F. Starr, and D. R. Smith, Science 314 (2006) 977.

[6] N. I. Landy, S. Sajuyigbe, J. J. Mock, D. R. Smith, and W. J. Padilla, Phys. Rev. Lett. 100 (2008) 207402. 
[7] J. B. Pendry, A. J. Holden, D. J. Robbin, and W. J. Stewart, IEEE Trans. Microwave Theory Tech. 47 (1999) 2075.

[8] H. Chen, L. Ran, J. Huangfu, X. Zhang, K. Chen, T. M. Grzegorczyk, and J. A. Kong, Phys. Rev. E70 (2004) 057605.

[9] V. A. Podlovsk, A. K. Sarychev, and V. M. Shalaev, Opt. Express 11 (2003) 735.

[10] J. Zhou, T. Koschny, L. Zhang, G. Tuttle, and C. M. Soukoulis, Appl. Phys. Lett. 88 (2006) 221103.

[11] V. D. Lam, J. B. Kim, N. T. Tung, S. J. Lee, Y. P. Lee, and J. Y. Rhee, Opt. Express 16 (2008) 5934.

[12] V. D. Lam, J. B. Kim, S. J. Lee, Y. P. Lee, and J. Y. Rhee, Opt. Express 15 (2007) 16651.

[13] V. D. Lam and Y. P. Lee, Advances in Natural Sciences 9 (2008) 117.

[14] Na Liu, Hongcang Guo, Liwei Fu, Stefan Kaiser, Heinz Schweizer, and Harald Giessen, Adv. Mater. 19 (2007) 3628.

[15] L. Jylha, I. Kolmakov, S. Maslovski, and S. Tretyakov, J. Appl. Phys. 99 (2006) 043102.

[16] M. Kafesaki, I. Tsiapa, N. Katsarakis, Th. Koschny, C. M . Soukoulis, and E. N. Economou, Phys. Rev. B75 (2007) 235114.

[17] E. Ozbay and K. Aydin, Photonics Nanostruct.: Fundam. Appl. 6 (2008) 108.

[18] V. D. Lam, J. B. Kim, S. J. Lee, and Y. P. Lee, J. Appl. Phys. 103 (2008) 033107.

[19] T. Koshny, M. Kafesaki, E. N. Economou, and C. M. Soukoulis, Phys. Rev. Lett. 93 (2004) 107402.

[20] A. MacKinnon and B. Kramer, Phys. Rev. Lett. 47 (1981) 1546.

[21] J. B. Pendry and A. MacKinnon, Phys. Rev. Lett. 69 (1992) 2772.

[22] J. B. Pendry, A. MacKinnon, and P. J. Roberts, Proc. R. Soc. London, Ser. A 437 (1992) 67

[23] J. B. Pendry, J. Mod. Opt. 41 (1994) 209.

[24] P. Markos and C. M. Soukoulis, Phys. Rev. B65 (2001) 033401.

[25] P. Markos and C. M. Soukoulis, Phys. Rev. E65 (2002) 036622.

[26] P. M. Bell, J. B. Pendry, L. M. Moreno, and A. J. Ward, Comput. Phys. Commun. 85 (1995) 306.

[27] J. Zhou, E. N. Economon, T. Koschny, and C. M. Soukoulis, Opt. Lett. 31 (2006) 3620.

[28] J. B. Pendry, A. J. Holden, W. J. Stewart, and I. Youngs, Phys. Rev. Lett. 76 (1996) 4773.

Received 11 December 2009. 